\title{
Balloon Valvuloplasty via the Pulmonary Artery Trunk for Treating Neonates With Severe Pulmonary Valve Disease
}

\author{
Kun-an Huang, Xiaofu Dai, MD, Dongshan, Liao, MD, Qianzhen Li, MD \\ Department of Cardiac Surgery, Fujian Medical University Union Hospital, Fujian, China
}

\section{ABSTRACT}

Background: Percutaneous balloon pulmonary valvuloplasty has proven to be a standard of care for neonates with severe pulmonary valve disease. However, the peripheral vessel injury, tricuspid chordae tendineae rupture, and cardiac tamponade could occur. Recently, we performed balloon valvuloplasty through pulmonary artery trunk. To date, the obtained outcome was promising.

Methods: Between January 2018 and December 2018, three neonates with critical pulmonary stenosis and two with membranous pulmonary atresia with intact ventricular septum were enrolled in our center. Balloon valvuloplasty through pulmonary artery trunk was performed in all patients. A 2-cm parasternal incision was made in the left third intercostal space. A guidewire was used to advance or perforate the pulmonary valve from the pulmonary artery trunk into the right ventricle, followed by balloon dilation of the valve.

Results: The procedure was successful in all patients. The oxygen saturation increased immediately after the balloon dilation, while the right ventricular systolic pressure and the gradient across the pulmonary valve decreased. No severe complications occurred.

Conclusions: Balloon valvuloplasty through pulmonary artery trunk is a safe and feasible alternative procedure. Thus, it could serve as a supplementary choice for treating severe pulmonary valve disease.

\section{INTRODUCTION}

Critical pulmonary stenosis (CPS) and pulmonary atresia with intact ventricular septum (PA/IVS) in neonates are characterized by ductus-dependent pulmonary circulation. Typically, patients develop severe cyanosis and right heart dysfunction shortly after birth, and the natural mortality rate is high. Reportedly, the successful decompression of the right ventricle (RV) would usually result in biventricular circulation and long-term survival of patients with CPS or PA/IVS, which is not complicated by Ebstein's anomaly or extensive ventriculocoronary connections [Coles 1989]. Therefore, early valvular opening is suggested to favor the

Received October 25, 2020; accepted November 12, 2020.

Correspondence: Qianzhen Li, 29 Xinquan Road, Gulou District, Fuzhou City, Fujian Province, China, 350001; 0086-13960756257 (e-mail:doctorlqz@163.com). pulmonary flow and the development of the RV. Since the first percutaneous balloon pulmonary valvuloplasty (PBPV) was reported by Kan et al. [Kan 1982] in 1982, the method has been applied as a standard of care for neonates with CPS or membranous PA/IVS with fewer complications [Loureiro 2017; McCrindle 1991]. However, several disadvantages, such as peripheral vessel injury, tricuspid chordae tendineae rupture, and cardiac tamponade caused by the perforation of the right atrial, right ventricular (RV), and pulmonary artery are noted. Even in an experienced center, these complications might occur in patients. In addition, excellent interventional skills from long-term training are needed. Besides, the percutaneous procedure requires a hybrid operation room and a specific wire for laser perforation or radiofrequency perforation, which is not cost-efficient. Although these conditions are easy to satisfy in some centers, the same is difficult in other centers, especially in developing countries.

Previously, we reported an alternative approach, wherein balloon pulmonary valvuloplasty (BPV) under transesophageal echocardiography (TEE) was performed via RV (RVBPV) after lower sternotomy [Lin 2017; Li 2013]. This technique is safe, simple, and effective. Recently, we performed BPV via pulmonary artery trunk (PAT-BPV), and the outcome was promising.

\section{MATERIALS AND METHODS}

Between January and December 2018, three patients with CPS and two patients with membranous PA/IVS were enrolled in our center. The morphology and hemodynamic data were detected by echocardiography. The diameters of the TV annulus (TVA) and PV annulus (PVA) were measured (Figure 1A, B). (Figure 1) The z-value was defined as follows: (measured diameter - mean normal diameter) / (standard deviation of mean normal diameter). Pressure measurements, including RV systolic pressure (RVSP) and peak pressure gradient across the PVA, were collected (Figure 1C). The exclusion criteria included a monopartite $\mathrm{RV}$, coronary fistulae with RV-dependent coronary circulation or concomitant with other complex congenital heart diseases. Patients with TV or PV z-value $<-5$ were excluded from the study. The characteristics of the patient are listed in Table 1. All patients required intervention within 30 days after birth to favor the pulmonary blood flow and RV development.

The patients were placed in the supine position after general anesthesia. A small transverse incision of about $2 \mathrm{~cm}$ 
Table 1. Characters of patients

\begin{tabular}{lccccc}
\hline Pt No./Dx & 1/PS & 2/PA & $3 / \mathrm{PS}$ & $4 / \mathrm{PS}$ & $5 / \mathrm{PA}$ \\
\hline Age (days) & 27 & 5 & 25 & 19 & 8 \\
Gender & Male & Female & Male & Female & Male \\
Weight $(\mathrm{kg})$ & 4.5 & 2.9 & 4.2 & 3.5 & 3.5 \\
PVA & & & & & \\
Diameter (mm) & 10.0 & 5.5 & 7.2 & 8 & 6.9 \\
Z value & 0.72 & -2.4 & -1.1 & -0.3 & -1.2 \\
TVA & & & & & \\
Diameter (mm) & 17 & 7.5 & 16 & 17 & 11 \\
Z value & 1.7 & -2.1 & 1.6 & 2 & -0.5 \\
\hline
\end{tabular}

PS, pulmonary stenosis; PA, pulmonary atresia with intact ventricle septum;

PVA, pulmonary valve annular; TVA, tricuspid valve annular

was made in the third left intercostal space. The lungs were deflated for 10-15 seconds, and the pericardium was opened and retracted to expose the pulmonary artery trunk (Figure 2A). Then, the ventilation of the left lung was restored. A U-like suture was placed on the surface of the pulmonary artery trunk (PAT), which was $1.5-2 \mathrm{~cm}$ from the PVA. A dose of $1 \mathrm{mg} / \mathrm{kg}$ heparin intravenously was administered, and the activated clotting time was confirmed to be $>250 \mathrm{~s}$. A trocar $(18 \mathrm{G})$ was punctured into the PAT at the location of the suture. Under the continuous instruction of TEE, the direction of the trocar was adjusted toward the PVA. In the case of PA/IVS, the trocar was guided to perforate into the middle of the pulmonary valve in a vertical direction. After withdrawing the needle, a guidewire was inserted into the trocar (Figure 2B). In the case of CPS, the guidewire was inserted and passed through the PVA under TEE guidance. Subsequently, the trocar was withdrawn, and a balloon (Tyshak-II, NuMed Canada Inc. Cornwall, Canada) with $3 \mathrm{~cm}$ length and 1.2-1.4fold of PVA diameter at width was advanced over the guidewire. The end of the balloon was advanced through the PVA for about $1.5 \mathrm{~cm}$ to ensure that the PVA was in the middle of the balloon (Figure 2D). The balloon was inflated manually with normal saline until the waist created by the pulmonary valve was eliminated for about 3s (Figure $2 \mathrm{E}$ ). This step was repeated three times. After balloon valvotomy, the hemodynamics were reassessed (Figure 2F). If the peak pulmonary valvular pressure gradient (PVPG) was still > $40 \mathrm{mmHg}$, a larger balloon was replaced, and the above steps were repeated. Subsequently, the balloon and guidewire were withdrawn. The puncture of the PAT was closed by securing the previously placed suture. A modified Blalock-Taussig shunt (B-T shunt) and ligation of the patent ductus arteriosus (PDA) or PDA stent implantation via the pulmonary artery might be required in case the peripheral oxygen saturation $\left(\mathrm{SpO}_{2}\right)$ was $<80 \%$ after balloon dilation. The incision was closed in a routine procedure with or without drainage (Figure 2C). PGE1 and milrinone infusions were continued until the successful establishment of ventricular pulmonary continuity.
Table 2. Clinical data in perioperative period and follow up

\begin{tabular}{|c|c|c|c|c|c|}
\hline Pt No./Dx & $1 / \mathrm{PS}$ & $2 / \mathrm{PA}$ & $3 / \mathrm{PS}$ & 4/PS & $5 / \mathrm{PA}$ \\
\hline Balloon size & 11 & 6 & 10 & 10 & 9 \\
\hline Volume of drainage & 25 & 30 & 25 & 35 & 30 \\
\hline Ventilation time $(\mathrm{h})$ & 20 & 13 & 8 & 12 & 10 \\
\hline Hospital stay (days) & 10 & 8 & 7 & 12 & 8 \\
\hline $\mathrm{F} / \mathrm{U}$ time (months) & 17 & 13 & 12 & 9 & 5 \\
\hline \multicolumn{6}{|l|}{ Gradient (mmHg) } \\
\hline Pre & 93 & - & 103 & 125 & - \\
\hline Post & 18 & 45 & 26 & 28 & 27 \\
\hline $\mathrm{F} / \mathrm{U}$ & 16 & 40 & 16 & 22 & 25 \\
\hline \multicolumn{6}{|l|}{$\operatorname{RSVP}(\mathrm{mmHg})$} \\
\hline Pre & 122 & 118 & 102 & 98 & 120 \\
\hline Post & 80 & 75 & 45 & 40 & 50 \\
\hline $\mathrm{F} / \mathrm{U}$ & 40 & 50 & 30 & 30 & 40 \\
\hline \multicolumn{6}{|l|}{ SPO2 (\%) } \\
\hline Pre & 60 & 70 & 79 & 81 & 75 \\
\hline Post & 95 & 88 & 87 & 89 & 90 \\
\hline $\mathrm{F} / \mathrm{U}$ & 96 & 96 & 96 & 95 & 96 \\
\hline \multicolumn{6}{|l|}{ TV regurgitation } \\
\hline Post & Mild & Mild & Mild & None & Mild \\
\hline $\mathrm{F} / \mathrm{U}$ & Mild & Mild & Mild & None & Mild \\
\hline \multicolumn{6}{|l|}{$\mathrm{PV}$ regurgitation } \\
\hline Post & Mild & Mild & Mild & Mild & Moderate \\
\hline$F / U$ & Mild & Mild & Mild & Mild & Moderate \\
\hline
\end{tabular}

PS, pulmonary stenosis; PA, pulmonary atresia with intact ventricle septum; F/U, follow up; Gradient, peak pulmonary valvular pressure gradient; Pre, preoperative; Post, postoperative; RVSP, right ventricular systolic pressure; $\mathrm{SpO}_{2}$, peripheral oxygen saturation; TV, tricuspid valve; $\mathrm{PV}$, pulmonary valve

All procedural or postprocedural complications, such as respiratory or cardiac arrest, pericardial effusion, arrhythmia or transfusion caused by bleeding, were recorded. The follow up was conducted at the outpatient service. All patients underwent echocardiography in the intensive care unit (ICU) and at follow up in order to determine the peak PVPG the degree of TV and $\mathrm{PV}$ insufficiency, and $\mathrm{SpO}_{2}$ levels.

\section{RESULTS}

The procedure was successfully performed in all patients. No B-T or PDA stent implantation was required. No pericardial effusion or cardiac tamponade was observed. No transfusion was needed. Also, no severe complications were detected. All patients were discharged uneventfully. The balloon size, the preoperative, and postoperative data of peak PVPG and RVSP, $\mathrm{SpO}_{2}$, mechanical ventilation time, and hospital stay 


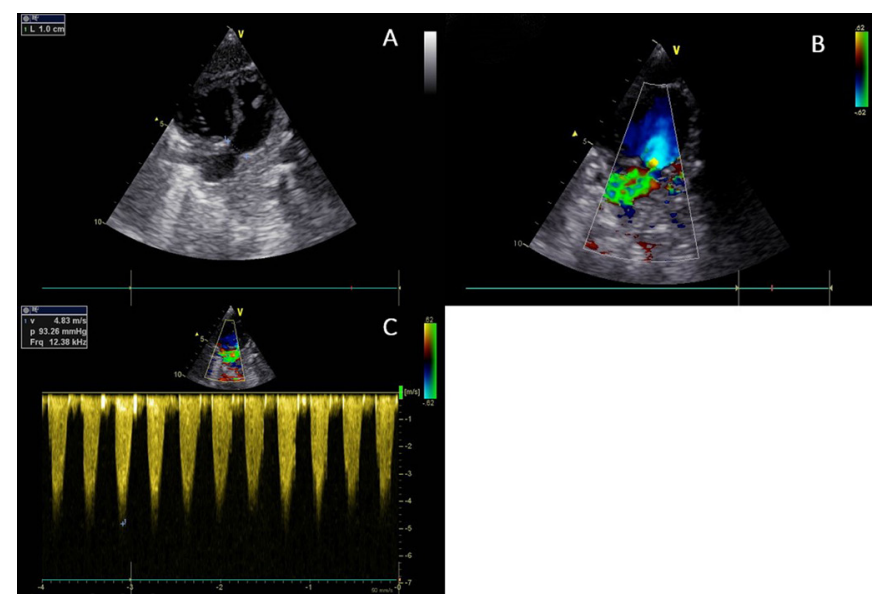

Figure 1. A, Pulmonary annular diameter of patient No. 1. B, The colorful jet across the pulmonary valve. C, The peak pulmonary valvular pressure gradient of patient No. 1.

are listed in Table 2. No severe TV and PV regurgitation was found in these cases. Also, none of the cases presented arrhythmia, cardiac perforation, main pulmonary artery aneurysm, or low output syndrome.

The last follow-up time was 6-17 months. All patients were alive. No secondary intervention was required. There were no cases of arrhythmia, hypoxia, or low output syndrome. The peak PVPG and RVSP, $\mathrm{SpO}_{2}$, and the degree of TV regurgitation, and PV regurgitation are listed in Table 2. No severe TV and PV regurgitation was found in these cases.

\section{DISCUSSION}

Different from PBPV, the track of PAT-BPV is PAT-PV$\mathrm{RV}$, which is shorter and avoids injury of the periphery vessels, TV, or TV chordae. The perforation is accurate, and it could be facilitated by a common trocar, but not a special wire. This procedure was monitored by TEE, which does not require $\mathrm{X}$-ray and hybrid operation room. Interestingly, it provides a straight direction and a proximal position for the wire. Then, the placement of the catheter and the perforation of PV becomes easier. Doctors do not require long-term training, which might decrease the consumption of medical resources, and thus, be an optimal alternative approach in deprived regions.

PAT-BPV approach has a similar effect to that of RV-PBV approach for treating CPS and membranous PA/IVS. Furthermore, it has additional benefits. First, sternotomy could be avoided, resulting in less pain and sternal malformation postoperative.

Second, according to our experience, it is easier to reach the PV via PAT as compared to that via RV. This phenomenon could be attributed to the thick outlet of RV in this cohort of patients, which in turn, causes the puncture of the RV and the controlling of the trocar's direction more difficult via RV than via PAT. Also, implant a PDA stent via PAT is rather simple.

Nevertheless, the present study has some limitations. Another incision is required for the additional B-T shunt once it is required
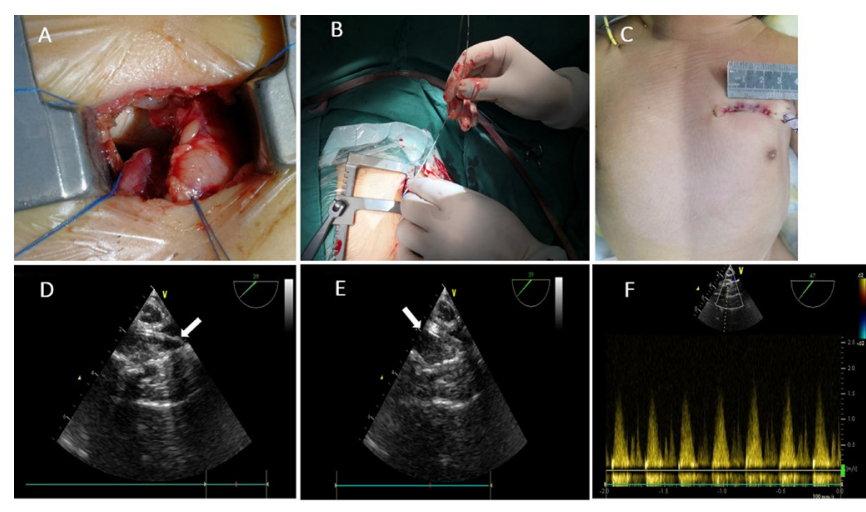

Figure 2. A, The pericardium was opened and retracted to expose the pulmonary artery trunk. B, A guidewire was inserted into the trocar through the pulmonary artery trunk. $C$, The incision was closed with or without drainage. The length of the incision was $2 \mathrm{~cm}$. D, The half of the balloon was placed in the pulmonary artery trunk and half in the right ventricle. $\mathrm{E}$, The balloon was dilated. F, The peak pulmonary valvular pressure gradient of patient No. 1 was measured after dilation.

after $\mathrm{BPV}\left(\mathrm{SpO}_{2}\right.$ less than $\left.80 \%\right)$. Currently, we did not perform the B-T shunt on the patients because of the small number of patients. Therefore, we could not deduce which is better-the stent implantation in PDA or the B-T shunt. Moreover, this single-center study necessitated a long-term follow up.

\section{CONCLUSION}

PAT-BPV is a simple, safe, and effective approach. It could avoid sternotomy and has fewer complications. Moreover, it can be easily mastered, leading to less consumption of medical resources, thereby serving as a supplementary choice for treating CPS or membranous PA/IVS.

\section{REFERENCES}

Coles JG, Freedom RM, Lightfoot NE, et al. 1989. Long-term results in neonates with pulmonary atresia and intact ventricular septum. Ann Thorac Surg. 47:213-7.

Kan JS, White RI, Jr., Mitchell SE, Gardner TJ. 1982. Percutaneous balloon valvuloplasty: a new method for treating congenital pulmonaryvalve stenosis. N Engl J Med. 307:540-2.

Li QZ, Cao H, Chen Q, Zhang GC, Chen LW, Chen DZ. 2013. Balloon valvuloplasty through the right ventricle: another treatment of pulmonary atresia with intact ventricular septum. Ann Thorac Surg. 95:1670-4.

Lin ZQ, Chen Q, Cao H, et al. 2017. Transthoracic Balloon Pulmonary Valvuloplasty for Treatment of Congenial Pulmonary Atresia Patients with Intact Ventricular Septum. Med Sci Monit. 23:4874-9.

Loureiro P, Cardoso B, Gomes IB, Martins JF, Pinto FF. 2017. Longterm results of percutaneous balloon valvuloplasty in neonatal critical pulmonary valve stenosis: a 20 -year, single-centre experience. Cardiol Young. 27:1314-22.

McCrindle BW, Kan JS. 1991. Long-term results after balloon pulmonary valvuloplasty. Circulation. 83:1915-22. 